\title{
Bauchfett führt eher zu Diabetes als Triglyzeride
}

\author{
Eine iranische Studie zeigt, dass viszerales Fett - abgelesen am Taillenumfang - eher mit \\ der Entwicklung eines Diabetes assoziiert ist als erhöhte Triglyzeridspiegel im Blut.
}

_ Subkutanes Fett spielt bei der Entwicklung eines Diabetes eine deutlich geringere Rolle als viszerales und besonders Leberfett. Auch die Triglyzeridlevel und die freien Fettsäuren im Blut spielen mit hinein. Es gibt also viele metabolische Phänotypen. Doch welche Kombination von Parametern hat das höchste Risiko? Eine iranische Studie vergleicht nun den Taillenumfang und erhöhte Triglyzeridwerte.

Unter den 3.500 Probanden der Isfahan Diabetes Prevention Study waren auch 1.865 erwachsene Nicht-Diabetiker, die einen Verwandten ersten Grades mit Typ-2-Diabetes hatten. Sie wurden regelmäßig oralen Glukosetoleranztests unterzogen. Zur Ermittlung des Diabetesrisikos wurden sie in Gruppen eingeteilt nach Taillenumfang (normal und erhöht) - als Surrogatparameter viszeraler Adipositas - sowie nach Triglyzeriden (erhöht, erniedrigt, normal).

Die logistische Regressionsanalyse ergab, dass bei erhöhtem Taillenumfang und erhöhten Triglyzeridwerten das
Diabetesrisiko 2,4-fach erhöht war. Personen mit normalem Taillenumfang und erhöhten Triglyzeridspiegeln hatten ein 1,9-fach erhöhtes Risiko. Der Clou: Personen mit erhöhtem Taillenumfang, aber normalen Triglyzeriden hatten ein 2,8-fach erhöhtes Risiko. - Janghorbani M, Amini M. Utility of hypertriglyceridemic
waist phenotype for predicting incident type 2 diabetes: The
Isfahan Diabetes Prevention Study. J Diabetes Investig 2016,
online 21. März; doi: 10.1111/jdi.12520

\section{KOMMENTAR}

Auch in der Bevölkerung des Mittleren Ostens bedeutet mehr Bauchfett mehr Diabetes. Unweigerlich stellt sich die Frage nach den Pathomechanismen. Es sind die gleichen wie hierzulande. Für den Taillenumfang gelten die internationalen Kriterien (Mann oberhalb von $102 \mathrm{~cm}$, Frau oberhalb $88 \mathrm{~cm}$ ). Ist der Taillenumfang größer, ist das Diabetesrisiko doppelt bis dreifach erhöht.

Wir wissen heute, dass über 200 Hormone aus dem viszeralen Fett stammen. Es ist vermutlich das größte endokrine Organ in unserem Körper. Diese Hormone sind mit prokoagulatorischen Prozessen, mit zentraler
Wirkung im Gehirn, v. a. aber mit metabolischen Erkrankungen assoziiert. Je mehr viszerales Fett da ist, desto mehr dieser Hormone werden produziert und desto höher ist wohl das Diabetesrisiko. Vermutlich gilt aber auch der Umkehrschluss - ist das Fett weg, ist auch der Diabetes weg.

Körperliche Aktivität ist das Beste, um es los zu werden. Da dieses Fett so viele Hormone produziert, ist es stoffwechselaktiver als subkutanes Fett und kann durch gesteigerte Verbrauchsprozesse gut reduziert werden.

Prof. Dr. med. P. Schwarz, MBA

\section{Kleine Korrektur: Ejakulationen und Prostatakarzinom}

In MMW 15/2016 hat sich auf Seite $39 \mathrm{im}$ Beitrag „Häufige Ejakulationen senken das Prostatakarzinom-Risiko" ein kleiner Fehler eingeschlichen. Als Studienergebnis wurde referiert, dass Männer mit 4-7 Ejakulationen pro Monat ein um $19 \%$ niedrigeres Risiko für ein Prostatakarzinom trügen als Männer mit $\geq 21$ Ejakulationen/Monat. Tatsächlich ist es natürlich anders herum.

\section{Schmerzhafte Blasen an beiden Händen}

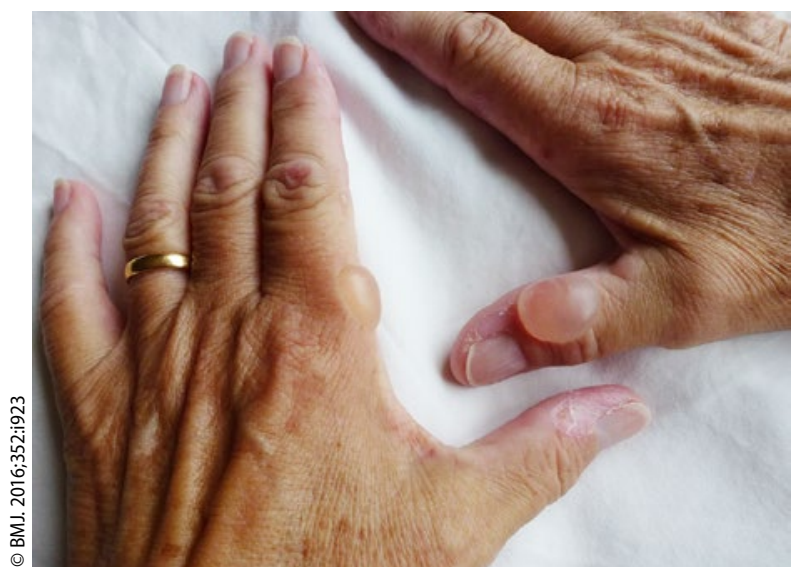

Blasenbildung an den Handrücken beidseits.
Eine 64-jährige Frau litt seit zwei Jahren an schmerzhafter Blasenbildung an den Handrücken beidseits. Eine topische Behandlung mit Steroiden besserte den Zustand nicht. Hautbiopsie, Urin- und Blutuntersuchungen bestätigten schließlich die Diagnose einer Porphyria cutanea tarda.

Diese ungewöhnliche Ursache einer Blasenbildung an den Händen tritt mit einer Häufigkeit von 1:200.000 auf und wird nicht selten verkannt. Differenzialdiagnostisch kommen ein bullöses Pemphigoid, ein dyshidrotisches Ekzem mit Ausbildung eines Pompholyx und eine Kontaktallergie in Betracht. An eine Porphyria cutanea tarda sollte man denken, wenn die Blasen an lichtexponierten Hautstellen auftreten und es an befallenen Stellen nebeneinander zu Blasenbildung, Erosionen, Hyperpigmentierung und Narbenbildung kommt. Der Urin ist zumeist dunkel gefärbt.

Prof. Dr. med. H. S. FüeßI

- Callander JA, Ponnambath N (jencallander@gmail.com). Porphyria cutanea tarda. BMJ. 2016;352:i923 